
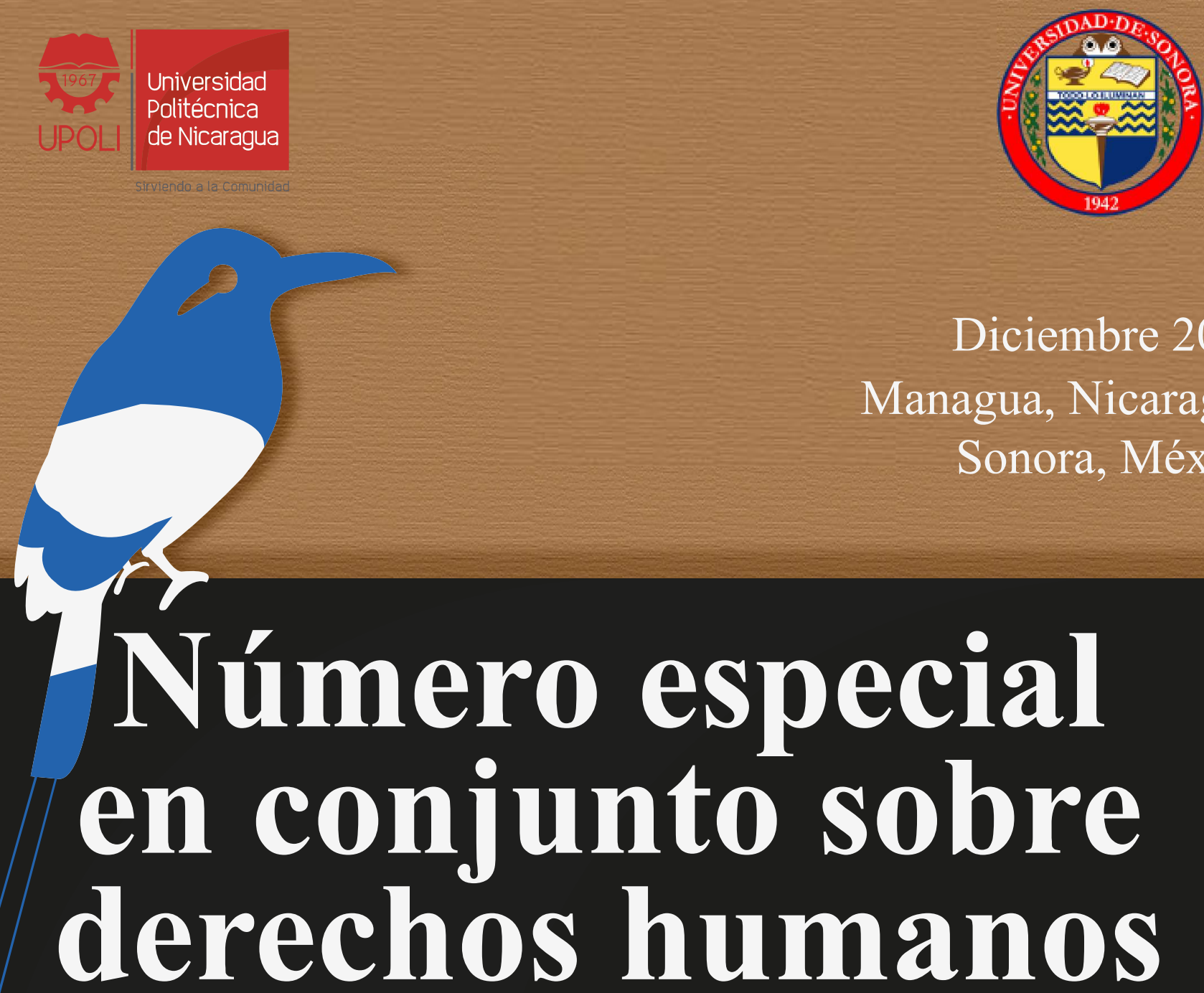

Cuaderno Jurídico y Político

e-ISSN: 2414-4428 ISSN: 2413-810X
BIOLEX

e-ISSN: 2007-5545

ISSN: 2007-5634
Revista Ciencia Jurídica y Política

e-ISSN: 2708-9266 ISSN: $2410-2768$

Sumario:

Presentación

Héctor Guillermo Campbell Araujo

Editorial

Óscar Castillo Guido

\title{
Reflexión académica
}

Tarsila González Camacho | Agustín Antonio Monreal Tirado

\section{Artículos}

Lorena Martínez Martínez | Margarita Cantero Ramírez | José Cruz Guzmán Díaz

Yuliesky Amador Echevarría

Guadalupe Aleida Valenzuela Miranda | Adria Velia González Beltrones | Rafael Ramírez Villaescusa

Reynaldo Jorge Lam Peña

Héctor Guillermo Campbell Araujo | Rosela de Jesús Rendón Rendón

Miguel Arturo Morales Zamorano |Guadalupe Aleida Valenzuela Miranda $\mid$ Rafael Ramírez Villaescusa

Christian Alberto Arellano López

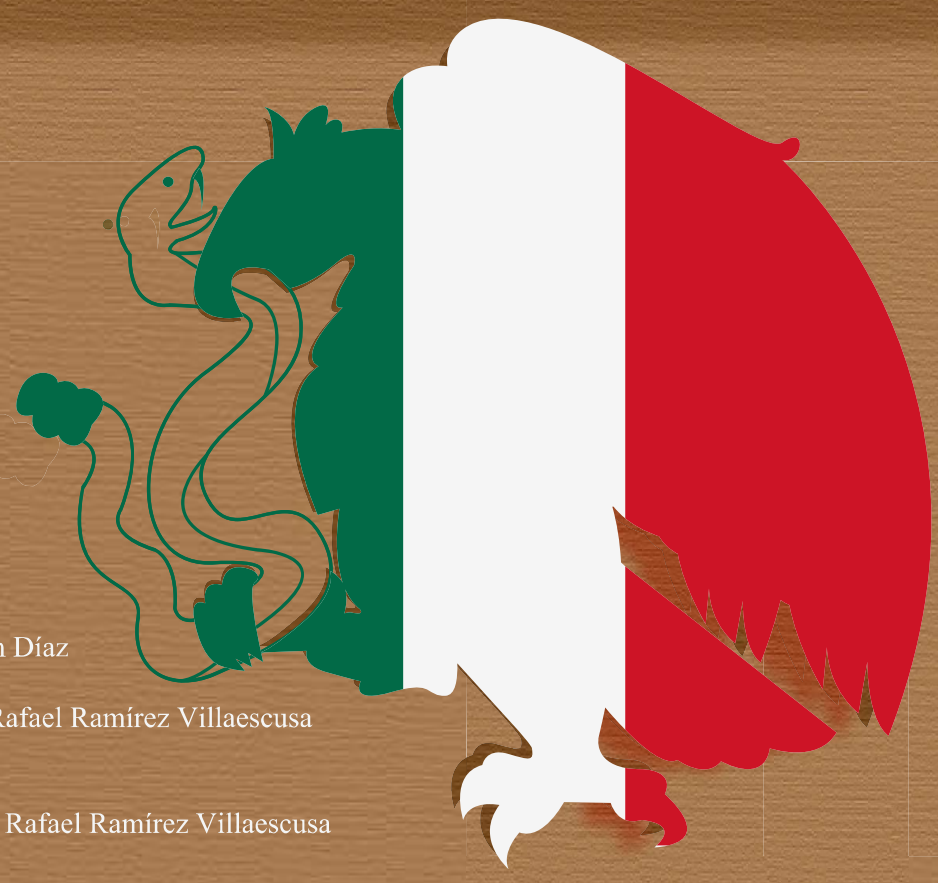




\section{DEFENSORÍA DE LOS DERECHOS HUMANOS EN EL ENTORNO UNIVERSITARIO \\ ESTUDIO DE CASO: UNIVERSIDAD DE SONORA, MÉXICO \\ DEFENDER OF HUMAN RIGHTS IN THE UNIVERSITY ENVIRONMENT \\ CASE STUDY: UNIVERSITY OF SONORA, MEXICO}

\section{Guadalupe Aleida Valenzuela}

Docente Investigadora del Departamento de Derecho de la Universidad de Sonora, México. Integrante del Cuerpo Académico "Derecho, Economía, Educación e Instituciones" CA-205UNISON.Grado Consolidado. Perfil PRODEP. SEP, correos.

Contacto: aleida.valenzuela@unison.mx (b) https://orcid.org/0000-0001-8052-0847

\author{
Adria Velia González Beltrones \\ Docente Investigadora del Departamento de \\ Derecho de la Universidad de Sonora, México. \\ Integrante del Cuerpo Académico "Derecho, \\ Economía, Educación e Instituciones" CA-205- \\ UNISON.Grado Consolidado. Perfil PRODEP. \\ SEP. \\ Contacto: adria.gonzalez@unison.mx \\ (D) https://orcid.org/0000-0001-6158-4458
}

\section{Rafael Ramírez Villaescusa}

Docente Investigador del Departamento de Derecho de la Universidad de Sonora, México. Líder del Cuerpo Académico "Derecho, Economía, Educación e Instituciones" CA205-UNISON.Grado Consolidado. Perfil PRODEP.

Contacto: rafael.ramirez@unison.mx

(1D) https://orcid.org/0000-0001-8138-498X

DOI https://doi.org/10.36796/biolex.v0i23.188

Recibido: 30.10.2020/Aceptado: 17.11 .2020

RESUMEN

El presente artículo aborda la importancia de la defensoría de los derechos humanos y sus implicaciones en el entorno universitario. Se planteó como objetivo explorar el nivel de información, y conocimiento sobre los derechos humanos en un sector de estudiantes de la Universidad de Sonora, México.

PALABRAS CLAVE

Derechos, defensoría, educación, humanos, superior.

ABSTRACT

This article addresses the importance of the human rights ombudsman and its implications in the university environment. The objective was to explore the level of information and knowledge about human rights in a group of students from the University of Sonora, Mexico.

Rights, defensoría, education, human, highe.

KEYWORDS 


\section{Sumario}

Introducción | Antecedentes | Revisión de la literatura | Metodología | Conclusiones| Referencias bibliográficas

\section{Introducción}

$\mathrm{E}$ n virtud del progreso de los derechos humanos previsto en la Constitución Política de los Estados Unidos Mexicanos, con lo que se armoniza la norma universitaria en el contexto constitucional, si bien su principal enfoque se centra en el ámbito de protección de los derechos universitarios. Resulta una oportunidad para realizar una investigación acerca de la importancia de la defensoría de los derechos humanos en el entorno universitario y su abordaje como órgano defensor.

Con esta reforma constitucional en materia de derechos humanos, constituye otro elemento fundamental para repensar la temática en la Universidad de Sonora, y abonar a los principios de imparcialidad y buena fe, para lo cual deberá promover acciones de prevención, difusión y consolidación de una cultura de paz en la comunidad universitaria, constituyéndose como órgano garante de los derechos humanos. Para seguir manteniendo la fortaleza de dicha institución es necesario hacer un reconocimiento de sus logros y una proyección crítica de su futuro.

Desde esta perspectiva, la cultura de derechos humanos desde las universidades nos obliga a replantear el alcance de sus competencias, lo que nos permitiría consolidar una cultura de convivencia armónica en comunidad y una clara conciencia de los universitarios como sujetos de derechos y deberes, en aras de ir formando una nueva ciudadanía que permitirá contribuir al proceso de consolidación del estudiante como ciudadano en su actuar cotidiano dentro de nuestra sociedad actual.

En este sentido, se establece en la mayoría de las universidades en el respectivo Programa Institucional de Derechos Universitarios, o en sus homólogos, como un instrumento sustantivo para el acompañamiento académicos y humano de los estudiantes, con el objetivo de regular los conflictos universitarios, establecer posibles orientaciones, particularmente en materia de violaciones de derechos humanos.

Desde este enfoque se pretende privilegiar la mediación y la conciliación como alternativas para dirimir conflictos en los asuntos de derechos humanos, de tal modo que pueda interactuar como órgano de consulta respecto de los casos que, en el ámbito de competencia, la comunidad universitaria someta a consideración 
a fin de contar con una opinión sobre la posible vulneración de los derechos humanos de los ciudadanos universitarios.

Entre los objetivos prioritarios se pretende atender más apropiadamente a los estudiantes que tengan conflictos en asuntos de derechos humanos, a través de diversas estrategias de mediación y conciliación.

Estrategias:

a) Se enfoca la intervención de los integrantes de la comunidad universitaria en la gestión de sus conflictos, mediante el uso de mecanismos alternativos de solución de controversias.

b) Se facilita la mediación y la conciliación en los casos en que los solicitantes tengan libre disposición de los derechos en conflicto, siempre que no se contravenga alguna disposición legal o se afecten derechos de terceros.

c) En el caso de violencia de género, se pretende que la mediación y conciliación procederá únicamente cuando la persona ofendida lo solicite, cuando cuente con el acompañamiento necesario para decidir participar en dicho proceso, y cuando reciba la información sobre los alcances derivados de tal participación.

d) Se orienta en los conceptos de buena fe, honestidad, equidad, flexibilidad, celeridad, gratuidad, legalidad, especialización, imparcialidad, información, veracidad, neutralidad y voluntariedad en la gestión de los conflictos.

e) Se vislumbra la participación como mediadores y conciliadores de los miembros de la comunidad universitaria que no formen parte de los órganos colegiados de gobierno, o bien autoridades administrativas, y cuenten con los conocimientos, habilidades, valores y conductas que el caso amerite.

Con los aspectos anteriores se destaca la pertinencia y necesidad de desarrollar acciones encaminadas a fortalecer los derechos humanos universitarios, impulsar la cultura y establecer obligaciones con las instituciones de educación superior. 


\section{Antecedentes}

El origen, de las defensorías universitarias, puede ser encontrado en el modelo del Ombudsman, escandinavo en el Defensor del Pueblo español ${ }^{1}$ En el ámbito universitario, a partir de la Ley de Reforma Universitaria de 1918, aparece hasta 1985 la primera Defensoría en la Universidad en nuestro país, la Universidad Nacional Autónoma de México, (UNAM) fue la primera en establecer la figura del Defensor de los Derechos Universitarios. Más tarde, surge en la Universidad Veracruzana, la Defensoría de los Derechos Universitarios, fue creada por Acuerdo de Rectoría de 6 de octubre de 2006, aprobado por el $\mathrm{H}$. Consejo Universitario General el 23 de noviembre del mismo año.

Un antecedente lejano de la DDU es la institución de la Procuraduría de Pobres establecida en la república mexicana en 1847, mediante una ley promovida por Ponciano Arriaga en el Estado de San Luis Potosí, México. Esta procuraduría inspiró a muchas de las Defensorías del Pueblo alrededor del mundo, aun cuando la mayoría seguiría principalmente el modelo escandinavo de ombudsman.

El antecedente más antiguo data del año de 1713 cuando se creó el Högste Ombudsmannen en Suecia, a raíz de una decisión del Rey Carlos XII de Suecia, quien deseaba contar con alguien que proteja sus intereses y controle la administración del Estado y a sus funcionarios.

Posteriormente se dio a mediados del siglo XX cuando se establecieron los University Ombudsman (Defensor del Pueblo Universitario) o Ombudsperson Council (Consejo del Defensor), en algunas instituciones terciarias de los EE. UU. La primera fue la Eastern Montana College en 1966, a la que siguieron un año después la Michigan State University, la Universidad Estatal de San Diego 1968, la Universidad de Cornell 1969, la Washington State University y la Universidad de Miami 1970. En la actualidad existen en más de doscientas instituciones de Estados Unidos y Canadá, entre otras.

Como ya se mencionó, la primera Defensoría de los Derechos Universitarios DDU en Iberoamérica, fue creada el 29 de mayo de 1985 en la Universidad Nacional Autónoma de México UNAM a propuesta del entonces rector Dr. Jorge Carpizo. Esta DDU cumple funciones similares a una defensoría del pueblo dentro de la UNAM, por lo que en México terminó inspirando la creación de un organismo

\footnotetext{
${ }^{1}$ Sánchez Castañeda, Alfredo y Daniel Márquez. La Defensoría de los Derechos Universitarios: Institución Original, Que Necesita Reformarse. (Colección Los Derechos Universitarios en el siglo XXI). México: Universidad Nacional Autónoma de México, 2016. Pág. 3.
} 
nacional, la Comisión Nacional de los Derechos Humanos, así como a las defensorías del pueblo.

Hoy en día la mayoría de las DDU mexicanas se congrega en la Red de Defensores, Procuradores y Titulares de Organismos de Defensa de los Derechos Universitarios. Numerosas universidades mexicanas públicas y privadas crearon sus propias defensorías en las últimas décadas. Nuestra Universidad de Sonora la incorporó formalmente el 15 de marzo de 2007 con el nombre de Comisión de Derechos Universitarios $\mathrm{CDU}^{2}$, asimismo, la idea trascendió a otros países de Latinoamérica, como Argentina, Bolivia, Brasil, Colombia, Ecuador, El Salvador, Honduras, Panamá y Perú, sin embargo, pese a la creciente difusión, todavía hay países sin casos de instituciones terciarias que no hayan incorporado la figura.

En Europa, además de España, muchos países cuentan con defensorías y redes, como Alemania, Austria, Bélgica, Francia, Italia y Gran Bretaña. Así mismo, se ha creado la Organización Europea de los Ombudsman de la Educación Superior.

Revisión de la literatura

La orientación conceptual del término de los derechos humanos retoma la definición oficial de la Organización de Naciones Unidas, que a la letra dice "son derechos inherentes a todos los seres humanos, sin distinción alguna de raza, sexo, nacionalidad, origen étnico, lengua, religión o cualquier otra condición. Entre los derechos humanos se incluyen el derecho a la vida y a la libertad; a no estar sometido ni a esclavitud ni a torturas; a la libertad de opinión y de expresión; a la

\footnotetext{
2 Vide: 1) Estatuto de la Comisión de Derechos Universitarios pp.2-3 [en línea] Disponible en: https://cdu.unison.mx/wp-content/uploads/2019/10/estatuto_cdu.pdf

2) Exposición de motivos del Reglamento Interno de la CDU[en línea] Disponible en: https://cdu.unison.mx/wpcontent/uploads/2019/06/reglamento_interno_cdu.pdf

La creación de la Comisión de Derechos Universitarios tiene su antecedente institucional más inmediato en los Planes de Desarrollo Institucional (PDI), correspondientes a los períodos 2001- 2005 y 2005-2009; el proyecto de su creación se materializa tras diversas iniciativas del sector académico, las cuales fueron llevadas a una Asamblea General de Trabajadores del Sindicato de Personal Académico de la Universidad de Sonora (STAUS) y aprobada por el Consejo General de Delegados. En el convenio de Revisión Salarial del 2005, la autoridad universitaria y el STAUS se comprometen a elaborar un proyecto que concrete la creación del Órgano de Defensa de los Derechos Universitarios y se crea una Comisión Mixta de Derechos Universitarios, de la cual emana el Estatuto de la Comisión de Derechos Universitarios, misma que es aprobada por el Colegio Académico el 15 de marzo de 2007.El presente reglamento, representa el primer esfuerzo de la Comisión de Derechos Universitarios, concebida como una instancia de buena fe cuyo interés se centra en la aplicación irrestricta de la normatividad universitaria para que los derechos por ella reconocidos se hagan efectivos a los miembros de la comunidad universitaria, los cuales pueden ser vulnerados por actos, resoluciones u omisiones de funcionarios, dependencias o cualquier otra entidad académica o administrativa, e inclusive, por cualquier miembro de la comunidad universitaria, porque dichos actos, resoluciones u omisiones sean irrazonables, injustos, inadecuados, erróneos o hayan dejado sin respuesta las solicitudes respectivas dentro de un plazo razonable, considerando las disposiciones establecidas para cada caso.
} 
educación y al trabajo, entre otros muchos. Estos derechos corresponden a todas las personas, sin discriminación alguna”. ${ }^{3}$

En otras palabras, los desafíos actuales y las propuestas apuntan, de manera preferente, en buscar el adecuado equilibrio entre la integralidad y la articulación de las temáticas referidas a la educación de los derechos humanos. ${ }^{4}$ Dado que los Derechos Humanos facilitan un marco ético y moral para lograr una convivencia armónica en una comunidad o sociedad, pues brindan lineamientos y criterios para que los individuos se respeten unos a otros y puedan evaluar sus relaciones con los demás. Así, los derechos humanos pueden ser entendidos como principios que organizan y guían las relaciones sociales y ayudan a construir una sociedad más justa, más inclusiva y menos violenta.

La Declaración Universal de los Derechos Humanos ha cumplido sesenta años de enunciada y asumida por la mayoría de las sociedades. Al cabo del tiempo, los Derechos Humanos se han convertido en una forma de cultura moral, un horizonte crítico que permite el cuestionamiento de aquellas prácticas que lesionan el cuerpo, la dignidad o las libertades de las personas en el mundo. Desde la elaboración de la propuesta en la que se reconocen raíces liberales, se pretendió que los principios presentes en la Declaración tradujeran un consenso intercultural en materia de protección de los seres humanos. ${ }^{5}$

El rol en los derechos humanos con relación al término que se discute se le conoce como el defensor del pueblo o bien, alto comisionado de las Cortes Generales encargado de defender los derechos fundamentales y las libertades públicas de los ciudadanos mediante la supervisión de la actividad de las administraciones públicas. Es decir, una autoridad del Estado encargada de garantizar los derechos de los habitantes ante abusos que puedan hacer los poderes políticos, en su caso, legislativo de ese mismo Estado. Desde el enfoque universitario se conoce como un órgano independiente cuya función consiste en tutelar y procurar el respeto de los derechos que la legislación otorga a los miembros de la comunidad universitaria. Funge como responsable de recibir quejas, realizar las investigaciones necesarias ante violaciones de los derechos universitarios y humanos dentro de la Universidad.

La figura del defensor cuenta con referencias remotas, algunos autores como Magendzo que debaten en un contexto político, económico, social y en ocasiones

\footnotetext{
${ }^{3}$ Organización de las Naciones Unidas. ONU. ¿Cómo promueve y protege la ONU los derechos humanos? 2020.

${ }^{4}$ Abraham Magendzo, K. La educación en derechos humanos en América latina: una mirada de fin de siglo.1999. Pág. 1.

${ }^{5}$ Gamio, Gehri Gonzalo. Ética, ciudadanía y derechos humanos. Pontificia Universidad Católica del Perú. 2020. Revista de Derecho. Pág. 174.
} 
analizan, el panorama de la educación en derechos humanos, como han mencionado especialistas en la materia, de reconocido prestigio, Carpizo, Humberto Nogueira Alcalá, Germán J. Bidart quienes ofrecen una definición clara, para entender la dignidad de la persona humana, con libertad y la igualdad como principios básicos que se van a concretar en derechos humanos. Autores como José Ovalle Favela, analiza "la reforma de 2011 al artículo 1o. de la Constitución Política, en la que se hace la distinción entre los derechos humanos y las garantías para su protección, señala que los derechos humanos tienen una doble fuente: la propia Constitución y los tratados internacionales de los que el Estado mexicano sea parte, y se establecen como principios para la interpretación de las normas sobre derechos humanos".

En este sentido la defensoría de los derechos humanos universitarios consiste en la figura del defensor universitario o procurador, se define como la autoridad principal en la mayoría de los casos, mismo que suele contar con defensores adjuntos o con visitadores, más el personal técnico y administrativo necesario. Hasta aquí hemos definido la facultad de este órgano de consulta para dirimir conflictos en los asuntos de derechos humanos.

En este tenor el Programa de la Organización de las Naciones Unidas para la Educación la Ciencia y la Cultura UNESCO, la nueva Agenda para el Desarrollo Sostenible 2030 representa un importante paso adelante en el reconocimiento de la contribución de la ciencia, los derechos humanos y el desarrollo sostenible. Hacer que la cultura desempeñe un papel central en las políticas de desarrollo no sólo constituye una inversión esencial en el futuro de nuestro mundo, sino que además es una condición previa para llevar a cabo con éxito procesos de mundialización que tengan en cuenta el principio de la diversidad cultural. Entre sus objetivos que se afiancen valores y principios universales como la solidaridad mundial, la inclusión, la lucha contra la discriminación, la igualdad de género y la rendición de cuentas.?

\section{Metodología}

La Metodología, en referencia al procedimiento utilizado para alcanzar el objetivo de la investigación, fue un estudio exploratorio para identificar la problemática relativa a los derechos humanos y el grado de conocimiento sobre el tema, de los estudiantes universitarios.

\footnotetext{
${ }^{6}$ José Ovalle Favela. Derechos humanos y garantías constitucionales. Boletín mexicano de derecho comparado versión On-line ISSN 2448-4873 vol.49 no.146 México may./ago. 2016

${ }^{7}$ UNESCO y los Objetivos de Desarrollo Sostenible,2030 [en lnea] disponible en:https://es.unesco.org/sdgs.
} 


\section{Materiales y técnicas}

Mediante una encuesta se exploró la información de los estudiantes sobre la importancia de los derechos humanos en el contexto universitario.

\section{Instrumento}

Para identificar el nivel de información se empleó una encuesta sencilla con 20 preguntas sobre los Derechos Humanos, incluyendo su definición, los principios fundamentales y la relación de los derechos humanos universitarios con el Estado, y su percepción en el contexto universitario.

La validación de las categorías se estableció por el consenso entre los profesores del área de investigación, y estudios en ciencias sociales, quienes individualmente analizaron su pertinencia y aplicación.

\section{Método}

Se contempló el Método analítico-sintético para estudiar las opiniones relacionadas entre sí respecto a las preguntas de la pesquisa. Se trata de una investigación cuantitativa ${ }^{8}$ tipo encuesta que busca identificar la temática, a través de un instrumento de valoración para establecer afirmaciones, que los participantes tienen sobre los derechos humanos. Dicho enfoque permite entender la forma en que los alumnos perciben la naturaleza de los derechos humanos.

\section{Participantes}

La muestra estuvo conformada por 240 estudiantes universitarios del segundo, quinto, y séptimo semestre de la licenciatura en Derecho, seleccionados con el criterio de haber estado inscritos en el semestre 2020-2. Todos participaron del estudio de manera voluntaria. Dado que la encuesta se colocó en la plataforma SIVEA, del portal Institucional considerando el razonamiento de la situación actual que viven los estudiantes ante la nueva modalidad virtual de la educación.

\section{Procedimiento}

Se procedió a contactar a los alumnos por correo electrónico a través de la plataforma institucional SIVEA y se les invitó a participar de manera voluntaria

\footnotetext{
${ }^{8}$ Hernández, Sampieri Roberto, Fernández Collado Carlos y Baptista Lucio Pilar. Metodología de la investigación. 2019. México: Mc Graw Hill.
} 
en dicha encuesta. Previamente a los participantes se les informó de la investigación. La encuesta se realizó en el mes de octubre de 2020.

\section{Resultados y discusión}

En lo referente a los resultados conjuntos de la muestra, debemos indicar que el $99.2 \%$ expresa conocer el significado de los derechos humanos; mientras que el $82.7 \%$ declara estar al tanto del rol de las universidades en materia de derechos humanos; un $90.3 \%$ señala conocer a quien protegen los derechos humanos; un 73.6\% marca conocer los principios de los derechos humanos, un $60 \%$ manifiestan que conocen algunos mecanismos para presentar una denuncia; un $85.5 \%$ conoce la responsabilidad administrativa e incluso penal; el $90.9 \%$ dice conocer los principales derechos humanos; un $87.2 \%$ conoce el debido proceso en materia de derechos humanos; mientras que un $42.1 \%$ desconoce alguna política para respetar los derechos humanos de los grupos en situaciones en vulnerabilidad.

Asimismo, se encontró que los estudiantes además de tener conocimiento sobre los derechos humanos conocen los principios, normativa, acuerdos, tratados, $\mathrm{y}$ convenios internacionales, suscritos por México, incluyendo la Declaración de los Derechos Humanos. Igualmente, manifiestan estar al tanto del rol de las universidades, la participación de los alumnos en discusiones, a través de mesas redondas, debates, charlas, talleres, foros en materia de derechos humanos.

La mayoría de los estudiantes encuestados afirman que existe una relación, en torno a este tema, con las actividades de reflexión que realizan sobre los derechos humanos. Otros señalan que han adquirido información de los profesores, como material de apoyo referente a los derechos humanos, para su estudio y discusión en clase. Asimismo, subrayan que si bien, esta temática no figura de manera transversal en los contenidos de todos los programas educativos del currículo, sí se le tiene contemplada como una asignatura optativa, (la cual escasamente los estudiantes seleccionan antes de alcanzar el número de créditos suficientes de la etapa del eje especializante) ${ }^{9}$

Existe un reconocimiento de la importancia de los derechos humanos para la protección, defensa, convivencia, respeto y vinculación de sus experiencias, en el ejercicio profesional de sus carreras. Discusión: se centró en saber los orígenes del resultado obtenido en el instrumento aplicado, sobre el grado de conocimiento

\footnotetext{
${ }^{9}$ La razón por la cual, esta asignatura es optativa y no obligatoria obedece a que el vigente Plan de la Licenciatura en Derecho (2004) es anterior a la Reforma Constitucional sobre Derechos Humanos en México (2011), por lo que dada su importancia y por la flexibilidad del Plan de estudios, permitió incorporarla en la lista de asignaturas optativas del eje especializante del currículo.
} 
sobre derechos humanos por parte de los estudiantes de los grupos seleccionados. Así, se encontró que, si bien la investigación exploratoria muestra una clara incidencia de los derechos humanos dentro de la formación profesional de los estudiantes de la licenciatura en derecho a estudio, ello obedece:

1. Más que a una política formal institucional, plasmada de forma obligatoria, en el currículo del vigente Plan de la Licenciatura en Derecho.

2. Al impulso de los docentes (a cargo de ciertas asignaturas, en las que su contenido se presta para relacionarlo, con esta importante reforma Constitucional sobre Derechos Humanos) para interesar a los estudiantes a su cargo, en la investigación y análisis de los DDHH, mediante el planteamiento de problemas locales, nacionales o internacionales relacionados con actos de autoridades que violentan, o no observan, en sus actuaciones los derechos humanos, cuyos resultados de tales investigaciones, con entusiasmo participan y las presentan en foros de discusión, seminarios locales, nacionales o internacionales.

Todo ello acorde al modelo educativo $2030,{ }^{10}$ que considera generar mejores y más condiciones para que los estudiantes de la Universidad de Sonora amplíen sus oportunidades y capacidades, bajo la perspectiva de desarrollo humano (Bedoya Abella, 2010), esto a través del fortalecimiento de dos ámbitos de la libertad como un aspecto de derechos humanos individuales: a) La generación de condiciones y oportunidades para que los estudiantes consigan sus objetivos más valiosos; b) La generación de condiciones y oportunidades para que los estudiantes ejerzan la libertad referida al papel primordial que tienen ellos en sus propios procesos de toma de decisión.

\section{Conclusiones}

Sin duda alguna nos encontramos en la época de los grandes cambios, principalmente en el campo de la educación superior, hacia una perspectiva 2030, según las directrices de la UNESCO. Los principios que orientan su camino parten de la identificación de los retos del entorno internacional y local, definiendo los componentes que se consideran necesarios para que la institución cumpla, de mejor manera, con el gran objetivo de formar profesionistas competentes, pero

10 Vide Modelo Educativo 2030 de la Universidad de Sonora, p.27, [en línea]Disponible en: https://www.unison.mx/institucional/marconormativo/reglamentosacademicos/MODELO_EDUCATIVO_2030.pd $\mathrm{f}$ 
también, ciudadanos participativos, respetuosos de los derechos humanos, y comprometidos con su defensa.

Los resultados de la intervención de los docentes de determinadas asignaturas, de incluir el tema de los derechos humanos, de manera transversal, indican que un estudio orientado de esta naturaleza, logra brindar información que ayuda a los estudiantes que ya tienen actitudes positivas a favor de los derechos humanos $\mathrm{y}$ a fortalecer sus argumentos, para incidir y tomar decisiones referidas a dicha problemática, sin embargo, por su naturaleza, es recomendable, de continuar la licenciatura en derecho con el vigente plan, incluirla como asignatura obligatoria del área vocacional, susceptible de lograr con ello un espectro mayor, o, en su caso, el contemplarla dentro de las asignaturas del área de formación integral y/o espacios educativos obligatorios compartidos del área básica entre los planes de estudio de licenciatura de la UNISON. ${ }^{11}$

Las nuevas tendencias representan una oportunidad de vanguardia y para ello es importante que las autoridades académicas se ocupen de incluirlas en los planes de estudio de sus carreras, de una manera estratégica, de tal modo que se considere el tema de los derechos humanos, como un eje prioritario, que requiere mayor compromiso por parte de la Institución.

Hoy en día es fundamental conocer sobre los Derechos Humanos 12 especialmente los deben conocer los estudiantes universitarios, puesto que, si son esenciales en la vida de cualquier persona, a ellos les corresponderá, desde sus espacios personales, profesionales o institucionales, fomentar su amplio desarrollo, garantizar la justicia, libertad de religión, de vivir en un ambiente sano, de forma igualitaria, de vivir saludables y plenos. Entre las razones que respaldan la importancia de la participación activa de los estudiantes tienen que ver con la profundización del conocimiento, reflexión, dominio, nociones básicas

\footnotetext{
${ }^{11}$ Vide Lineamientos para el Componente Curricular del Modelo Educativo 2030 de la Universidad De Sonora Lineamientos 4,5,6 y 49 [en línea] Disponible en: https://www.unison.mx/institucional/marconormativo/ reglamentosacademicos/Lineamientos_para_el_Componente_Curricular.pdf.

12 Vide ONU Los derechos humanos son un tema transversal en todas las políticas y los programas de la ONU en los ámbitos clave sobre desarrollo, asistencia humanitaria, paz y seguridad, así como en asuntos económicos y sociales. Por ello, prácticamente todo órgano de la ONU y organismo especializado se ve envuelto de alguna forma en la protección de los derechos humanos. Algunos ejemplos de ello son el derecho al desarrollo, que es la base de los Objetivos de Desarrollo Sostenible; el derecho a la alimentación, defendido por la Organización de las Naciones Unidas para la Alimentación y la Agricultura; los derechos laborales, defendidos y protegidos por la Organización Internacional del Trabajo; la igualdad de género, proclamada por ONU Mujeres; los derechos del niño, de los pueblos indígenas y de las personas con discapacidad. El Día de los Derechos Humanos se celebra todos los años el 10 de diciembre. [en línea] Disponible en: https://undocs.org/Home/Mobile?FinalSymbol= A\%2FRES\%2F41\%2F128\&Language=S\&DeviceType=Desktop.
} 
del tema, y con acciones de sintonía, tutela y defensa de los derechos universitarios en sus aspectos, académicos, profesional y personal.

Para que los resultados del trabajo exploratorio descrito, no se quede únicamente en buenas intenciones, es necesario que las Instituciones de Educación Superior IES como nuestra Universidad de Sonora, de forma proactiva, apunten: a incluir, de manera estratégica, en sus Planes de estudio de licenciaturas, la asignatura de Derechos Humanos, a construir, una cultura de respeto por estos derechos, y, a educar para una nueva ciudadanía, con mayor comprensión acerca de los Derechos Humanos y la importancia de su ejercicio y aplicación para todos.

\section{Referencias bibliográficas}

Bayón Gamio, Gehri Gonzalo. Ética, ciudadanía y derechos humanos. Pontificia Universidad Católica del Perú. 2020. Revista de Derecho. themis 57. Recuperado de: Dialnet-EticaCiudadaniaYDerechosHumanos-5110476.pd

Declaración Universal de los Derechos Humanos. Recuperado de: http://www.jus.uio.no/lm/un.universal.declaration.of.human.rights.1948/portrait. a4.pdf

Hernández, Sampieri Roberto, Fernández Collado Carlos y Baptista Lucio Pilar. Metodología de la investigación. 2019. México: Mc Graw Hill.

Magendzo K. Abraham. La educación en derechos humanos en América latina: una mirada de fin de siglo.1999. Pág. 1. Recuperado de: http://www.derechoshumanos.unlp.edu.ar/assets/files/documentos/la-educacionen-derechos-humanos-en-america-latina-una-mirada-de-fin-de-siglo-abrahammagendzo.pdf. La escuela y los derechos humanos.2008. Recuperado de: http://www.seg.guanajuato.gob.mx/CEducativa/Maestros/Formacin\%20Continua \%20Maestros/Magendzo-Completo.pdf

Organización de Naciones Unidas. ONU. Derechos Humanos. Recuperado: 27/10/2020. https://www.onu.org.mx

Organización de Naciones Unidas. ONU. ¿Cómo promueve y protege la ONU los derechos humanos? 20/10/2020/. Recuperado de: https://www.un.org/es/sections/what-we-do/protect-human-rights/

Organización de las Naciones Unidas para la Educación, la Ciencia y la Cultura. Declaración Universal de los derechos Humanos.1948. Recuperado de: https://portal.unesco.org/es/ev.phpURL_ID=26053\&URL_DO=DO_TOPIC\&URL_ SECTION=201.html

Organización de las Naciones Unidas para la Educación, la Ciencia y la Cultura. Declaración mundial sobre la educación superior en el siglo XXI: visión y acción y marco de acción prioritaria para el cambio y el desarrollo de la educación 
superior. $1998 . \quad$ Recuperado de: http://www.unesco.org/education/educprog/wche/declaration_spa.htm\#declaraci on

Organización de las Naciones Unidas para la Educación, la Ciencia y la Cultura. Comunicado Mundial sobre la Educación Superior- 2009: La nueva dinámica de la educación superior y la investigación para el cambio social y el desarrollo. Recuperado de: http://www.unesco.org/education/WCHE2009/comunicado_es.pdf

Organización de las Naciones Unidas para la Educación, la Ciencia y la Cultura. La UNESCO y los Objetivos de Desarrollo Sostenible.2020. Recuperado de: https://es.unesco.org/sdgs

Sánchez Castañeda, Alfredo y Daniel Márquez. La Defensoría de los Derechos Universitarios: Institución Original, Que Necesita Reformarse. (Colección Los Derechos Universitarios en el siglo XXI). México: Universidad Nacional Autónoma de México, 2016. pág. 3.

Vilchez Choquehuayta, Gissela y Frisancho Hidalgo, Susana. Creencias sobre los derechos humanos en estudiantes universitarios de la ciudad de Lima. Revista SciElO. Peru.vol.20 no.1 Lima ene. 2014. 



\begin{tabular}{l|l} 
ECJP & $\begin{array}{l}\text { Escuela de } \\
\text { Ciencias } \\
\text { Uurídicas y } \\
\text { Políticas }\end{array}$
\end{tabular}

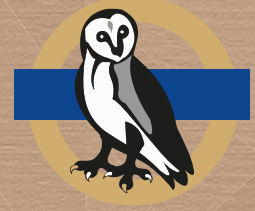

Cuaderno Jurídico y Político y Revista de Ciencias Jurídicas y Políticas son revistas académicas de publicación semestral del Instituto Centroamericano de Estudios Jurídicos y Políticos ICEJP y la Escuela de Ciencias Jurídicas y Políticas respectivamente.

BIOLEX Revista Jurídica del Departamento de Derecho es una publicación semestral por la Universidad de Sonora, a través de la División de Ciencias Sociales, Departamento de derecho.

BIOLEX incorporada en:
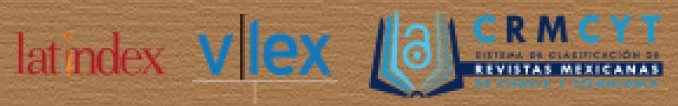

División de Ciencias Sociales

Universidad de Sonore, URC

CJP y RevistaCJP incorporada en:

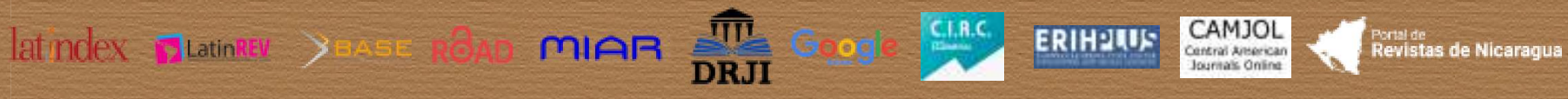

